\title{
INTERFACE CRACK PROPAGATION IN POROUS AND TIME-DEPENDENT MATERIALS ANALYZED WITH DISCRETE MODELS
}

\author{
THEOCHARIS BAXEVANIS, FRÉDÉRIC DUFOUR, AND GILLES PIJAUDIER-CABOT
}

\begin{abstract}
A model describing the crack propagation at the interface between a rigid substratum and a beam is considered. The interface is modeled using a fiber bundle model (i.e. using a discrete set of elements having a random strength). The distribution of avalanches, defined as the distance over which the crack is propagated under a fixed force, is studied in order to capture the effect of ageing and time dependent response of the interface. The avalanches depend not only on the statistical distribution of strength but more importantly on time (or displacement) correlations. Namely, local fiber breakage kinetics is related to a correlation length, which sets the size of the fracture process zone which occurs ahead of the crack due to progressive failure. First, a variation of porosity of the interface is considered. It corresponds for instance to diffusion controlled dissolution processes. Interpreting the results in [11], it is shown that the size of the fracture process zone increases with increasing porosity in accordance with experimental observations [12]. The creep - fracture interaction is analyzed in the second part of the paper. It is found based on a Maxwell model that the size of the process zone depends on the fracture propagating velocity and on the distribution of forces in the interface due to the interaction between the interface and the rest of the specimen. The observed decrease of the size of the process zone, in creep experiments, compared to the size of the process zone in a static process, is justified by the proposed model for an interface that is less viscous than the rest of the material.
\end{abstract}

\section{INTRODUCTION}

Progressive failure of quasi-brittle heterogeneous materials is a succession of micro-cracks nucleation, propagation and arrest. First, the material response is elastic, then microcracking appears and eventually these microcracks coalesce in order to form a macro-crack which propagates suddenly. The above rupture events are controlled by the randomness of the distribution of the material properties and also by internal correlation lengths that are in general unknown. Such correlation lengths have a strong influence on the failure of quasi-brittle materials. Instead of a perfect crack, with a very small process zone of nonlinear response at its tip, a rather large fracture process zone (FPZ) develops in the material ahead of the tip of the macrocrack [8]. This process zone induces a size effect, which is typical of quasi-brittle heterogeneous structures. Among several possible explanations for such a size effect on the structural strength, a simple one is that it is a purely deterministic effect, resulting from the energetic interaction between the elastic part of the structure and the FPZ. From the point of view of design of structures, e.g. reinforced concrete structures, this size effect is important. According to the size effect law proposed by Bažant, the nominal strength of a structural component can be decreased by as much as 50 percent if the real size of the structure is five times larger than the laboratory specimen size. This size effect law incorporates also the definition of a fracture energy, seen as the energy dissipation in the asymptotic

Key words and phrases. Zip model, FPZ size, size effects, creep, ageing, fracture, viscoelasticity, time effect, concrete failure, discrete approach. 


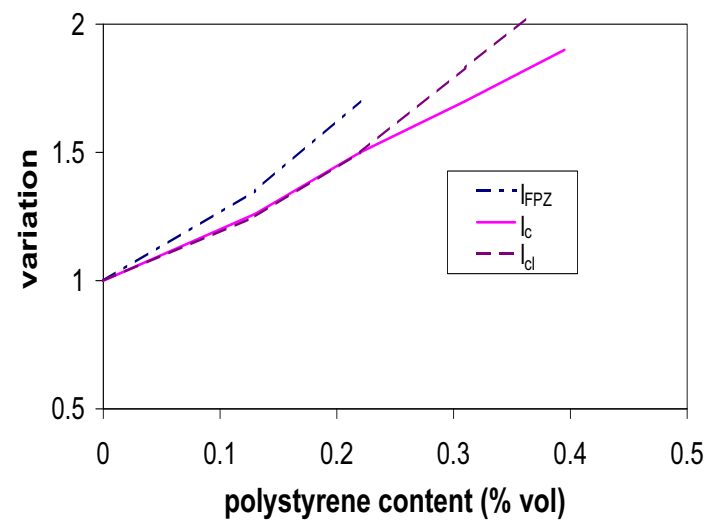

FIgURE 1. Evolution of the width of the FPZ measured experimentally $\left(l_{F P Z}\right)$, internal length $\left(l_{c}\right)$, and the Irwin's length $\left(l_{c l}\right)$ with polystyrene content in model materials.

limit of a structure of infinite size with a very large initial notch which would fail at crack initiation.

A relevant continuum model has to take into account this size effect, and at the same time to capture strain softening due to progressive cracking in a way that is physically and mathematically sound. This is usually performed by the introduction of an internal length in the continuum model (see e.g. the review in [4]). The internal length serves two purposes at the same time: (1) it is a way of constraining the energy dissipation to occur in a region of finite size upon strain localization due to softening and to keep the governing equations well posed [19], (2) the occurrence of the FPZ upon cracking and the inherited size effect are captured at the same time. As a matter of fact, size effect, e.g. of geometrically similar notched three point bending beams, is an indirect technique for the calibration of the internal length and other model parameters for isotropic non local damage models for concrete [15]. Theoretical strain localization analyses show that the width of the fracture process zone ought to be proportional to the internal length. Such a result was already observed numerically by Bažant et al [6] and used in order to design an approximate method for the determination of the internal length [7]. Experiments and finite element analyses on several model materials made with mix of mortar and polystyrene beads [12] have shown that the width of the FPZ and Irwin's length (which can be seen as the length of the FPZ ahead of the macrocrack tip) are also correlated to the variation of the internal length in the non local continuum. Figure (1) shows that the FPZ measured experimentally from acoustic events analysis during fracture, the internal length obtained after calibration of the non local damage model on the various model materials (using size effect test data) and Irwin's length correlate quite well. Irwin's length is defined as

$$
l_{c l}=\frac{E G_{f}}{\sigma^{2}}
$$

in this equation, $E$ is the Young's modulus, $G_{f}$ is the fracture energy and $\sigma$ is the tensile strength.

These experiments on model materials, with various amount of polystyrene beads, were designed in order to mimic the ageing process in cementitious materials due to calcium leaching, i.e. a progressive dissolution of the material due 


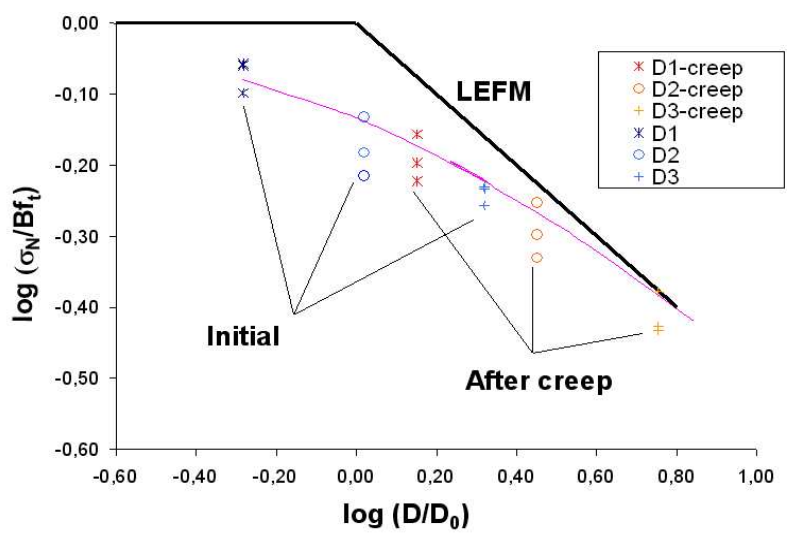

Figure 2. Evolution of size effect test data for 3 point bending tests on notched specimens subjected to creep prior fracture (after Pijaudier-Cabot et al. [20])

to contact with water. In this case, the width and the length of the FPZ increase with the amount of material porosity and can be considered as proportional to the internal length, at least in the three point bending beam tests on notched specimen considered in [12]. Furthermore, the same experimental study has shown that large variations of the fracture energy occur with the amount of polystyrene content. Such variations were also observed in fracture tests performed after accelerated leaching on mortar beams [16]. Without variations of the internal length, it was not possible to model the consequences of the leaching process on the fracture properties of aged materials, and in particular the variations of the size effect parameters observed by Le Bellégo et al [16] which indicate variations of the size of the FPZ.

There is another situation of structural response where similar variations of the size effect properties have been observed. It is the case of creep - fracture interaction studied in [3]. The authors conducted size effect tests on geometrically similar specimens loaded at different rates and observed that the slower the loading rate, the more brittle the response. Pijaudier-Cabot et al [20] performed a series of three point bending beam tests (notched specimens) with and without applying a sustained load for 3 months up to 85 percent of the maximum load prior to the fracture test. They arrived to a similar conclusion, namely that creep influences the residual capacity of the beams, but also that upon size effect tests, the specimens which were subjected to creep exhibited a more brittle response. This property yields a shift to the right of the data set on the size effect plot as shown in Figure (2). The same phenomena has been observed by Bažant and Gettu [3] and Bažant and Li [5] for linear creep.

In the present paper, we use a discrete random modeling based on a fiber bundle model in order to investigate the size of the FPZ. It is the so-called ZIP model developed in [11]. We consider the two examples depicted in the above paragraphs, namely the case of a material whose porosity increases and the case of creep fracture interaction. As we will see in section 2, it is quite straightforward to demonstrate that, according to the discrete model, when the microstructural size increases (in order to capture the heterogeneities in a representative volume) the FPZ length ought to increase too. The case of creep - fracture interaction needs additional mathematical considerations, but upon some simplifying assumptions, it will be shown in section 3 that the FPZ length decreases as creep develops. 


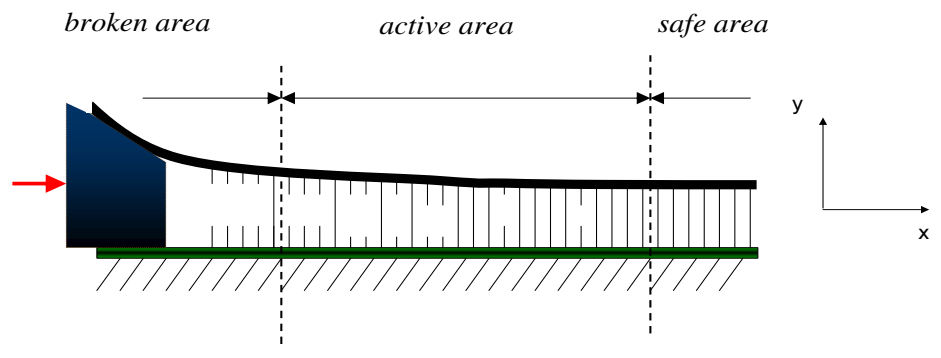

FIgURE 3. Schematic representation of "zip" model. The wedge moves along the $x$-axis.

\section{ZIP MODEL - CASE OF INTERFACE WITH VARIATION OF POROSITY}

During the past decades, fiber bundle models have received considerable attention and have been studied extensively. Originally introduced to explain ruptures in heterogeneous materials under tension [9], fiber bundle models have been applied to cracks and fractures, earthquakes, and other related breakdown phenomena [1] and [21]. They are directed towards the study of the material heterogeneities, i.e. at a scale much smaller than the representative volume of the material. These models consist of a set of parallel fibers having statistical distributed strength. The sample is loaded parallel to the fiber direction, and a fiber breaks if its elongation exceeds a threshold value. When a fiber breaks, its load is transferred to other surviving fibers in the bundle, according to a specific transfer rule. Among the possible options of load transfer are the assumption of equal load sharing (global-sharing rule) [9] and the much studied variants - local load-sharing rule- where the load on the failing fiber is distributed equally among the nearest surviving fibers [13]. There are also a number of studies that may be placed among the two extremes that global and local load-sharing rules constitute. Among them is the study by Delaplace et al [11], who constructed an hierarchically connected fiber bundle model. They have worked out analytically and numerically the statistics of avalanches in a system whose geometry mimics the propagation of crack front at the interface between a rigid support and an elastic beam. Other work on hierarchical fiber bundle models can be found in [18], [10] and [22]. For a review of the literature in the subject, one may refer for instance to the work of Batrouni et al [2].

In this section, we focus on a system of elastic-perfectly brittle fibers loaded in parallel between a rigid substratum and a semi-infinite elastic beam. The geometry is suited to an inhomogeneous, but steady state of loading and it can be considered as a schematic model for mode I crack propagation (the rigid side of the interface representing an axis of symmetry). The nonlinear interface serves to concentrate cracking onto a line, the same as in the cohesive crack model. A normal displacement is imposed at one point of the elastic beam that can move along the interface as if a wedge was pushed in a double cantilever geometry (Figure (3)). The fiber strength is randomly distributed with a uniform probability of critical fiber extension between 0 and 1 . As the wedge is moving along the $x$-axis, the interface decouples into three different parts due to the randomness of fiber strength. Namely, under the wedge and in a small area ahead all fibers are broken. This region constitutes the crack itself. Further ahead there is an active area. Moving along this zone, broken fibers become more and more scarce and their influence can be neglected. 
The exponential decay of fiber rupture ahead of the crack tip provides the means to define the size of the fracture process zone.

A simplified model where the displacement profile along the upper beam is imposed is used. The interface opening has an exponential shape: for any abscissa $x$, the profile $y$ is given by

$$
y(x)=\exp \left(\frac{U(t)-x}{\xi^{\prime}}\right)
$$

where the length scale

$$
\xi^{\prime}=\sqrt{2}(E I / k)^{1 / 4},
$$

is fixed ( $E$ is the Young modulus of the beam, $I$ its transverse geometrical inertia and $k$ the stiffness of the fibers divided by the spacing in between the fibers), and $U(t)$ is the time-dependent horizontal displacement of the wedge. As shown in [11], this simplified model has the same statistical properties as the one where an elastic beam is connected with elastic-perfectly brittle fibers to the rigid substratum. We are not going to derive again the complete results obtained by the authors of [11] who used analytical solutions and analyzed the distribution of avalanches. We will recall just the main useful results, and in particular the motivations for using a simplified approach, with a fixed beam profile (2) instead of a deformable one.

In the spirit of continuum modeling (i.e. for length scales much larger than the fiber separation) and using beam theory, one can write an equation for the mean deflection of the beam $y(x)$ (also fiber elongation), based on the survival uniform force that fibers apply onto the beam. This force for elastic fibers would be simply $k y(x)$.

where

$$
E I \frac{d^{4} y(x)}{d x^{4}}=-k y(x)(1-Y(x))
$$

$$
Y(x)=\max _{x^{\prime} \geq x}\left(y\left(x^{\prime}\right)\right) .
$$

This equation holds for a uniform distribution of critical fiber extension between 0 and 1 , and for $y<1$, whereas $d^{4} y(x) / d x^{4}=0$ for larger $y$. The boundary conditions are $y(\infty)=d y(\infty) / d x=0, y(0)=1$ and $d^{2} y(0) / d x^{2}=0$ (no bending moment applied at the loading point). The distinction between $y$ and $Y$ is made because damage is an irreversible process and thus one has to compute the maximum damage having been met by the corresponding section of fibers. $(1-Y(x))$ actually represents the probability of survival of fibers. There is no analytical solution to this problem. However, since the quadratic nonlinear term becomes unimportant at a large distance from the origin, the asymptotic shape will have the form

$$
y^{\prime}(x)=A e^{-x / \xi^{\prime}} \cos \left(x / \xi^{\prime}+\phi\right),
$$

where $\xi^{\prime}$ is defined in $(3), \phi$ is such that $\cos (\phi)=1 / A>0$ in order for $y^{\prime}$ to satisfy the boundary conditions, while $A$ is chosen such that

$$
\left.\frac{\partial y^{\prime}(x)}{\partial x}\right|_{x=0}=-\frac{1}{\xi^{\prime}}\left(1+A \sqrt{1-(1 / A)^{2}}\right)
$$

is equal to the corresponding value of the exact solution. The oscillatory component is the one that makes the deflection nonmonotonous and requires the distinction between $y$ and $Y$. Omitting this oscillatory term and the nonlinearity, one retrieves (2) which is found to yield a correct assumption compared to full numerical calculations with a deformable beam in the zip model [11].

A typical time variation of total force acting on the beam (see Fig ...) shows a constant mean value through time with large fluctuation though. There exists various definitions of avalanches. The one used in [11] is the most natural. It consists 


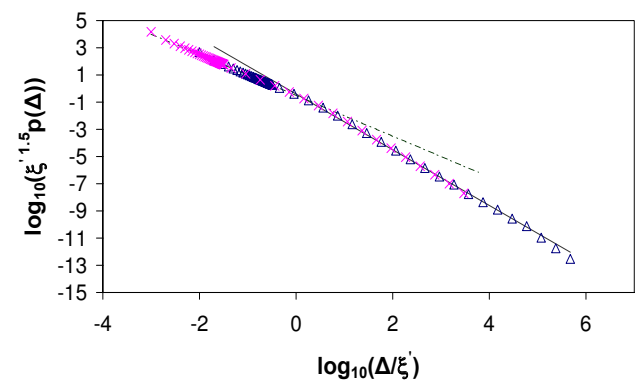

FIgURE 4. Avalanche distribution for the simplified model of imposed beam deformation (eqn (2)) for two different values of $\xi^{\prime}$, namely $\xi^{\prime}=100(\triangle)$ and $\xi^{\prime}=1000(\times)$. Scaled variable $\Delta / \xi^{\prime}$ and scaled distribution $\xi^{\prime 1.5} p(\Delta)$ are used. The dashed line is guideline with slope -1.5 ; continuous line is guideline with slope -2.05 .

in fixing a level of force, and computing the distance $\Delta U$ over which the crack can propagate which is the retrival of the force level. The smallest avalanche is 1 (fiber spacing) and the largest one can be in theory infinite when computed for a level of force corresponding to the maximum ever reached but with an infinitely small probability. The avalanches are characterized by their statistical distribution, $p(\Delta U, F)$. In order to give a global characterization of the signal without considering a specific value of the force, one may consider the above avalanches for any crack length $\Delta U$ such that a fiber would break, and then average over all breaking events. These avalanches are the "forward" avalanches as described in Fig .... In order to investigate the effect of $\xi^{\prime}$ on the size of the FPZ, we shall just consider that the avalanche distribution follows two regimes with respect to a crossover value $\Delta^{*}$ of the avalanche size $\Delta \equiv \Delta U$. In the first regime the statistical distribution of avalanches is a power law $p(\Delta) \propto \Delta^{\tau_{1}}$ with an exponent $\tau_{1}$. In this regime the force versus crack length displays correlations similar to a random walk $\left(\tau_{1} \simeq-1.5\right)$. In the second regime, the distribution of avalanches is again a power law but with a different exponent $\tau_{2}$. In this regime, it can be shown that the forces are uncorrelated $\left(\tau_{2} \simeq-2\right)$ (Figure (4)). The cross over value $\Delta^{*}$ scales the extent of the FPZ. Same as in Hillerborg's fictitious crack model [14], it is the length of the FPZ, i.e. the size beyond which fiber breakage is not influenced by the crack tip.

In Figure (4) the scaled variable $\Delta / \xi^{\prime}$ and the scaled distribution $\xi^{\prime 1.5} p(\Delta)$ were used to show that curves corresponding to different values of $\xi^{\prime}$ collapse onto one master curve. Hence, the size of the fracture process zone scales as $\xi^{\prime}$. More specifically the size of the FPZ increases as $\xi^{\prime}$ increases (Figure (5)).

The Young modulus $E$ of the beam is a positive, monotonically decreasing function $\mathbb{F}$ of porosity $g ; E=E_{0} \mathbb{F}(g)$, where $E_{0}$ is a reference value. Porosity $g$ here is defined as the ratio of the voids area to the total area of the beam. Furthermore, the ratio $k$ of the average stiffness of the interface is the same function: $k=k_{0} \mathbb{F}(g)$, where $k_{0}$ is a reference value. In the fracture process zone, however, it is not the average porosity of the interface that is of importance. In the $2 \mathrm{D}$ material that 


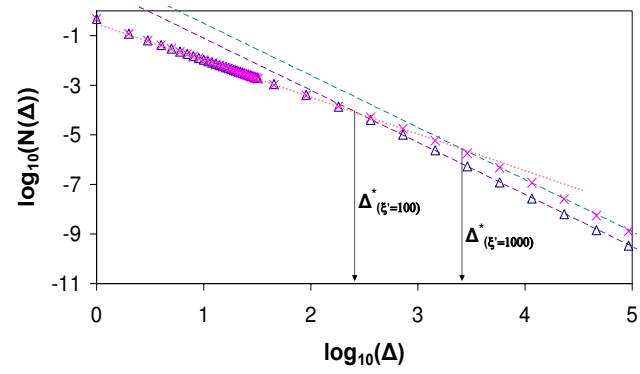

FIGURE 5. Avalanche distributions for the simplified model of imposed beam deformation (eqn (2)) for two different values of $\xi^{\prime}$, namely $\xi^{\prime}=100(\triangle)$ and $\xi^{\prime}=1000(\times)$. The size of the fracture process zone (crossover value) for $\xi^{\prime}=100$ is less than the respective size for $\xi^{\prime}=1000$. The dashed lines are guidelines with slope -2.05 and the dotted one with slope -1.5 , while the continuous ones mark the crossover values.

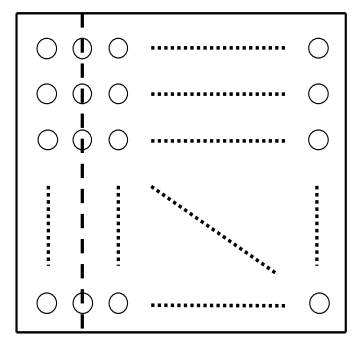

FIgURE 6. Schematic representation of a material as a square of edge $R$. The circles of radius $r$ in within represent $n^{2}$ uniformly distributed voids.

the zip model aims at describing, fracture follows the weakest possible path. Consequently, the porosity of the interface should be less than the average interface porosity as the crack connects the pores in the material in such a way that the work of fracture is minimum. In the case of a regular array of voids for instance, the interface porosity $g_{l}$ along the dotted line in Figure (6) is $g_{l}=n 2 r / R \propto \sqrt{g}$. One can generalize this remark and set that $g_{l}$ scales as $g^{b}$, where $b$ will be in principle a real number smaller than 1 . One can argue also that in the porous material, the roughness of the crack will increase, thus increasing the fracture energy. In the zip model, it would be equivalent to change the fiber spacing which is not sensitive on the avalanche statistics. Hence, submitting stiffnesses accounting for porosity 
into (3), one gets

$$
\xi^{\prime}=\sqrt{2}\left(\frac{E_{0} \mathbb{F}(g) I}{k_{0} \mathbb{F}\left(g^{b}\right)}\right)^{1 / 4} .
$$

Since $g<1$ and $\mathbb{F}$ is a positive, monotonically decreasing function of $g$, one can conclude that $\mathbb{F}(g) / \mathbb{F}\left(g^{b}\right)$ increases with increasing $g$. It is then obvious from (8) that increasing porosity yields an increase in $\xi^{\prime}$ and consequently an increase in the size of the FPZ. So the present theoretical model is consistent with experimental observations on the dependence of the size of FPZ on porosity, as well as with numerical computations with a non local damage model.

\section{Zip MODEL - CASE OF VISCOElastiC FIBERS LOADED BETWEen A Rigid SUBSTRATUM AND A VISCOELASTIC BEAM}

The model described below is a generalization in viscoelasticity of the previous model developed in [11], (Figure (3)). All fibers are viscoelastic, following a Maxwell model, and perfectly brittle (i.e. fail suddenly at a given elongation) with the same stiffness $k$ and viscous coefficient $\mu$. However, the strength of the fibers are random uncorrelated variables in order to incorporate inhomogeneity into the model, same as in the elastic case. The fibers are loaded in parallel between a rigid support and a semi-infinite viscoelastic beam of Young modulus $E$, transverse geometrical inertia $I$ and viscous coefficient $M$. One could as well consider, instead of the rigid part, another viscoelastic beam even with different properties without any change in the formulation of the problem and in the qualitative results that will be drawn eventually.

In notation, the position of the fiber $i$ is denoted as $x_{i}$ and its deformation as $y_{i}$. The strength of each fiber denoted as $y_{i}^{c r}$ is, as mentioned above, a random uncorrelated variable having a statistical distribution in the interval $[0,1]$. The spacing between the fibers is set to 1 (i.e. $x_{i+1}-x_{i}=1$ ), thus defining a fixed microstructural size. A normal displacement of 1 is imposed at one point of the beam, which may move along the interface, as if a wedge is pushed in a double cantilever geometry (Figure (3)).

The fiber deformation $y_{i}$ is related to force $F_{i}$ as

$$
\frac{\partial F_{i}(t)}{\partial t}+\frac{k}{\mu} F_{i}(t)=k \frac{\partial y_{i}(t)}{\partial t} \quad(\text { Maxwell model }),
$$

where $t$ denotes the time and $\partial$ denotes partial differentiation with respect to the indicated variable. It can also be shown without altering the assumptions of beam theory that the deformation of the beam $y(x, t)$ satisfies a similar equation, if again the Maxwell model is used,

$$
\frac{\partial F(x, t)}{\partial t}+\frac{E}{M} F(x, t)=E I \frac{\partial^{5} y(x, t)}{\partial t \partial x^{4}},
$$

where $F(x, t)$ denotes the force acting on the beam and the other parameters are already defined.

In the spirit of continuum modeling (i.e., for length scales much larger than the fiber separation) the system defining the mean deflection of the beam $y(x, t)$ reads:

$$
\left\{\begin{array}{l}
\partial F(x, t) / \partial t+\frac{E}{M} F(x, t)=E I \partial^{5} y(x, t) / \partial t \partial x^{4} \\
\partial F(x, t) / \partial t+\frac{k}{\mu} F(x, t)=-k \partial(y(x, t)(1-Y(x, t))) / \partial t
\end{array}\right.
$$

where

$$
Y(x, t)=\max _{t^{\prime} \leq t}\left(y\left(x, t^{\prime}\right)\right) .
$$


The above system holds for a uniform distribution of fiber extension between 0 and 1 , and for $y<1$, whereas $\partial^{4} y / \partial x^{4}=0$ for larger $y$. The boundary conditions are such that

$$
\begin{aligned}
\forall t, \quad y(\infty, t) & =\partial y(\infty, t) / \partial x=0, \partial^{2} y(U(t), t) / \partial x^{2}=0, \\
& \text { and for } t \geq t_{0}, \quad y(U(t), t)=1,
\end{aligned}
$$

where $U(t)$ denotes the time-dependent horizontal displacement of the wedge along the $x$-axis, $t_{0}$ is the time at which the wedge starts moving, and the relation $\partial^{2} y(U(t), t) / \partial x^{2}=0$ implies that no bending moment is being applied at the loading point, while the initial conditions are

$$
y(x, 0)=y_{0}(x) \text { and } F(x, 0)=F_{0}(x) .
$$

3.1. Simplified version of the model: constant displacement profile. Let us derive a simplified model, where the displacement profile along the upper beam is imposed, that serves for analytical study. For this purpose we neglect oscillatory and non linear term due to $Y$.??? This simplifying assumption will allow for closedform expressions. In the same spirit as in [11] (Eqns. (4)-(7)), the profile of the upper beam is derived, but now for the case of a viscoelastic beam connected to a viscoelastic interface, although the interface still fails for a sufficient elongation.

The governing equations read now

$$
\left\{\begin{array}{l}
\partial F(x, t) / \partial t+\frac{E}{M} F(x, t)=E I \partial^{5} y(x, t) / \partial t \partial x^{4} \\
\partial F(x, t) / \partial t+\frac{k}{\mu} F(x, t)=-k \partial y(x, t) / \partial t .
\end{array}\right.
$$

In notation, we denote the system (15) with boundary conditions (13) and initial conditions (14) as $\left(\Sigma^{*}\right)$.

We eliminate $F$ in (15) to obtain

$$
\frac{\partial}{\partial t}\left(E I \frac{\partial^{5} y(x, t)}{\partial t \partial x^{4}}+k \frac{\partial y(x, t)}{\partial t}\right)=-\frac{k}{\mu} E I \frac{\partial^{5} y(x, t)}{\partial t \partial x^{4}}-\frac{E}{M} k \frac{\partial y(x, t)}{\partial t} .
$$

By separation of variables, i.e. by assuming that

$$
\partial y(x, t) / \partial t=u(t) v(x)
$$

we take from (16)

$$
\frac{\partial u(t) / \partial t}{u(t)}=\frac{-\frac{k}{\mu} E I\left(\partial^{4} v(x) / \partial x^{4}\right)-\frac{E}{M} k v(x)}{E I\left(\partial^{4} v(x) / \partial x^{4}\right)+k v(x)}=\alpha,
$$

where $\alpha$ is a positive constant. Indeed, the displacement $y(x, t)$ is a monotonically increasing function and hence $u(t)$ and $\partial u(t) / \partial t$ are both positives. Solving the above equation for $u$ and $v$, and then integrating (17), we find a solution of system (15)

$$
\left\{\begin{array}{l}
y(x, t)=\frac{1}{\alpha} e^{-x / \xi} \cos (x / \xi) e^{\alpha t} \\
F(x, t)=\frac{k}{\frac{k}{\mu}+\alpha} e^{-x / \xi} \cos (x / \xi) e^{\alpha t},
\end{array}\right.
$$

where

$$
\xi=\sqrt{2}\left(\frac{E I}{k} \frac{\frac{k}{\mu}+\alpha}{\frac{E}{M}+\alpha}\right)^{1 / 4} .
$$

By multiplying in (19) with $\alpha$, taking into account the linearity of (15) (i.e. any linear combination of solutions is a solution itself), and omitting the oscillatory term $\cos (x / \xi)$, we end up to another solution of (15)

$$
\left\{\begin{array}{l}
y(x, t)=e^{(\alpha \xi t-x) / \xi} \\
F(x, t)=\frac{\alpha k}{\frac{k}{\mu}+\alpha} e^{(\alpha \xi t-x) / \xi} .
\end{array}\right.
$$




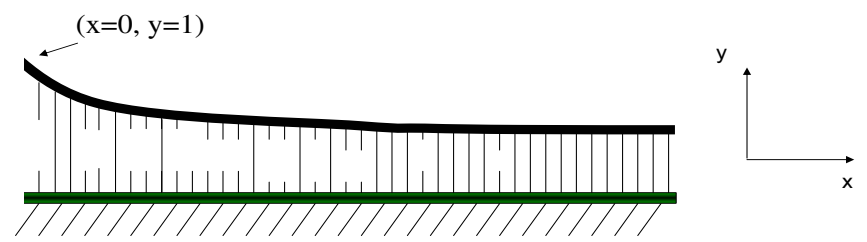

Figure 7. At time $t_{0}$, such that $e^{t_{0}}-1=\alpha$, the vertical displacement of the loading point is equal to 1 . The wedge now starts moving along the $x$-axis.

Further, for initial conditions

$$
\left(y(x, 0)=e^{-x / \xi}, F(x, 0)=\frac{\alpha k}{\frac{k}{\mu}+\alpha} e^{-x / \xi}\right),
$$

(21) is a solution of system $\left(\Sigma^{*}\right)$ for $t_{0}=0$. It represents the problem of the wedge moving along $x$-axis under constant velocity $v=\alpha \xi$, (Figure (3)). Indeed, for the deformation $y(x, t)$ at position $x$ to become equal to 1 ,

$$
y(x, t)=e^{(\alpha \xi t-x) / \xi}=1,
$$

time $t=x /(\alpha \xi)$ is needed.

It should be noted here that $\alpha$ is a parameter that characterizes the deformation rate. Although the deformation rate is not constant $\left(\partial y(x, t) / \partial t=e^{(\alpha \xi t-x) / \xi}\right)$ the process can be viewed as quasi-static since for constant $\xi$ the total force exerted on the wedge, which is the sum of all fiber contributions, remains fixed within the statistical randomness of the fiber strength.

Suppose now that the initial conditions are

$$
\left(y_{0}(x)=0, F_{0}(x)=0\right),
$$

then

$$
\left\{\begin{array}{l}
y(x, t)=\frac{1}{\alpha} e^{-x / \xi}\left(e^{\alpha t}-1\right) \\
F(x, t)=\frac{k}{\frac{k}{\mu}+\alpha} e^{-x / \xi}\left(e^{\alpha t}-1\right),
\end{array}\right.
$$

is one solution of system (15). For $t_{0}$, such that $e^{t_{0}}-1=\alpha$, the above equation (25) gives

$$
\left(y\left(x, t_{0}\right)=e^{-x / \xi}, F\left(x, t_{0}\right)=\frac{\alpha k}{\frac{k}{\mu}+\alpha} e^{-x / \xi}\right) .
$$

Hence, for $t \geq t_{0}$, solution (25) reads

$$
\left\{\begin{array}{l}
y(x, t)=e^{(U(t)-x) / \xi} \\
F(x, t)=\frac{\alpha k}{\frac{k}{\mu}+\alpha} e^{(U(t)-x) / \xi},
\end{array}\right.
$$

where $U(t)=\xi \ln \left(\frac{e^{\alpha t}-1}{\alpha}\right) . U(t)$ represents the time-horizontal displacement of the wedge for $t \geq t_{0}$ (Figure (7)). In this form it is apparent that (25) satisfies the boundary conditions (13). Thus (25) is a solution of $\left(\Sigma^{*}\right)$. 
Although the wedge does not move, in this case, under constant velocity, the process is still quasi-static since again the total force exerted on the wedge remains constant within the statistical randomness.

We showed above that, at least for two different initial conditions, namely for (22) and (24), the system $\left(\Sigma^{*}\right)$ admits an asymptotic solution of the form

$$
\left\{\begin{array}{l}
y(x, t)=e^{(U(t)-x) / \xi} \\
F(x, t)=\frac{\alpha k}{\frac{k}{\mu}+\alpha} e^{(U(t)-x) / \xi}, \quad(\text { see }(21) \text { and }(27)) .
\end{array}\right.
$$

These solutions represent the motion of a wedge whose time-dependent horizontal displacement for $t \geq t_{0}$ is $U(t)$ while before is at rest. To derive the above solutions (27), we omitted the oscillatory component $\cos (x / \xi)$ and made the assumption that a fiber breaks when it is strained up to its critical extension $y_{i}^{c r}$ which is statistically distributed in the interval $[0,1]$. Although from experimental evidence we know that in viscoelasticity the threshold cannot be placed in general neither to the total deformation nor to the elastic deformation. Indeed for low stress levels although creep strain can be large -even larger than that corresponding to peak stress for short term loading- there is no significant damage [17]). On the other hand, if one wishes to model the coupling between creep and damagen the threshold cannot be set only on the elastic part. Our assumption is justified by the fact that the loading path is identical for all fibers and thus they are expected to fail at the same total deformation. Indeed, the elastic deformation $y_{i}^{e l}$ of every fiber remains through out the process the same fraction of its total deformation $y_{i}^{\text {tot }}$

$$
y_{i}^{e l}(x, t)=\frac{\alpha k}{\frac{k}{\mu}+\alpha} y_{i}^{t o t}(x, t) .
$$

This situation corresponds to experimental material tests.

3.1.1. Avalanche distribution. As it is already pointed out, the model described here is the "viscoelastic" analog of the model in [11]. For matters of convenience, we refer to the model described here as "viscoelastic" model and to the one developed in [11] as "elastic" model. Looking now at the parameters $\xi(20)$ and $\xi^{\prime}(3)$ that scale the size of the fracture process zone in the two models respectively, we see that in the "viscoelastic" model $\xi$ depends not only on the stiffness fraction $E / \kappa$ as it is for the "elastic" model $\left(\xi^{\prime}\right)$ but on the relaxation times $\kappa / \mu$ and $E / M$ as well, and on the parameter $\alpha$ which is related to the rate of deformation. $\alpha$ may also be seen as a parameter that scales the fracture propagating velocity. Indeed, the time-horizontal displacement of the wedge $U(t)$ depends on $\alpha$ (see (21) and (27)) and in turn sets the fracture propagating velocity. Indeed Figure (8) shows that the average distance between the moving wedge and the crack tip remains constant, up to the randomness of the model, thoughout the total time of computations. Further, when both the interface and the rest of the material are elastic in the "limit" $(\mu, M \rightarrow+\infty)$, the "elastic" case can be reproduced, $\xi \rightarrow \xi^{\prime}$. This is also the case $\left(\xi \rightarrow \xi^{\prime}\right)$ when the rate of deformation is too large to allow for creep, i.e. when $\alpha \rightarrow+\infty$ or when the interaction between the interface and the rest of the material does not depend on their relaxation times, i.e. when $(k / \mu=E / M)$. $\xi$ can be either greater or less than $\xi^{\prime}$, with the two end members of the spectrum to be the case of an elastic beam and a viscoelastic interface and the case of a viscoelastic beam and an elastic interface (Figure (9)). The case of a beam with relaxation time $E / M$ greater than that $k / \mu$ of the interface corresponds to the observed decrease of the size of the fracture process zone in creep experiments compared to the size of the FPZ in a rate independent process. This should be the case, i.e. the composite should be more viscous than the interface, in order for our 


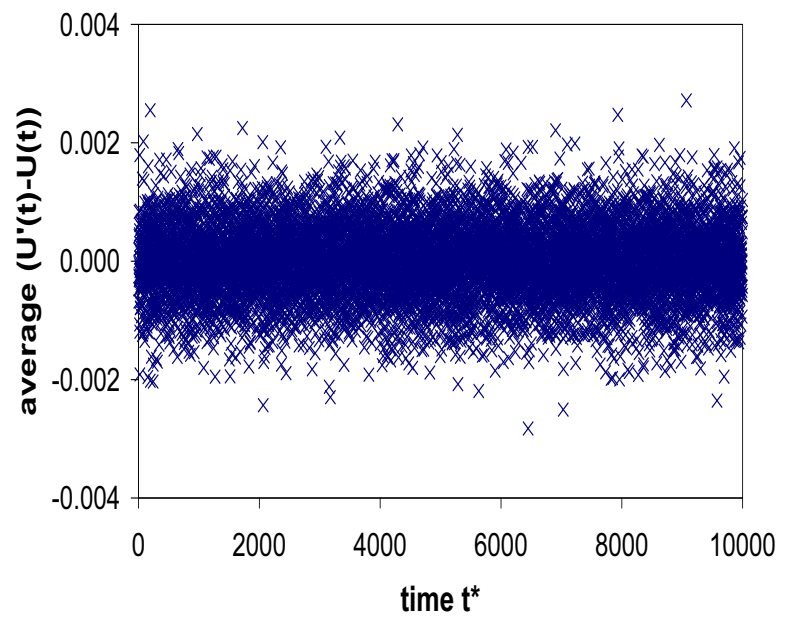

Figure 8. The evolution of the averages on the relative displacement of the crack tip, $U^{\prime}(t)$, minus the relative displacement of the wedge $U(t)$ in time intervals of 10000 broken fibers, is plotted, against time $t^{*}$, which is equal to the total time (which corresponds to $10^{8}$ broken fibers) divided by the length of the aforementioned time intervals. Since these averages remain through out the process very close to zero, the velocity of the crack tip coincides with the velocity of the wedge.

model to be realistic; in order for the fibers to remain alive when the matrix material in between has failed. The fibers may represent for example, based on the work of Hillerborg et al [14] who studied fracture in concrete with the help of a cohesive crack, the aggregates that bridge the crack and allow for stress transfer across the crack. The fiber bundle model developed in this paper mimics the propagation of the crack front at an interface which ahead of the crack tip collapses onto a line as in the cohesive crack model. The relaxation time of the aggregates is usually less than that of concrete.

3.2. Model for the complete problem. We now come back to the complete problem $(\Sigma)$, i.e. system (11) with boundary conditions (13) and initial conditions (14). Our aim is rather to compute the approximate average shape of the deformed beam. One can see that in system $(\Sigma)$ for the damaged interface the quadratic nonlinear term $\partial(y Y) / \partial t$, which is additional compared to system $\left(\Sigma^{*}\right)$ for the undamaged interface, becomes unimportant far away from the boundary $x=0$. Thus the asymptotic shape can have a similar expression as the one used in the previous section.

$$
\left\{\begin{array}{l}
y^{\prime}(x, t)=A(t) e^{-x / \xi} \cos \left(-\frac{x}{\xi}+\phi(t)\right) e^{\alpha t} \\
F^{\prime}(x, t)=\frac{\alpha k}{\frac{k}{\mu}+\alpha} A(t) e^{-x / \xi} \cos \left(-\frac{x}{\xi}+\phi(t)\right) e^{\alpha t}
\end{array}\right.
$$

where $\phi$ is such that $\cos (\phi)=1 / A>0$ in order for the solution $y^{\prime}$ to satisfy the boundary condition $y^{\prime}(0, t)=e^{\alpha t}$, while $A$ is chosen such that

$$
\left.\frac{\partial y^{\prime}(x, t)}{\partial x}\right|_{x=0}=-\frac{1}{\xi}\left(1+A(t) \sqrt{1-(1 / A(t))^{2}}\right) e^{\alpha t}
$$




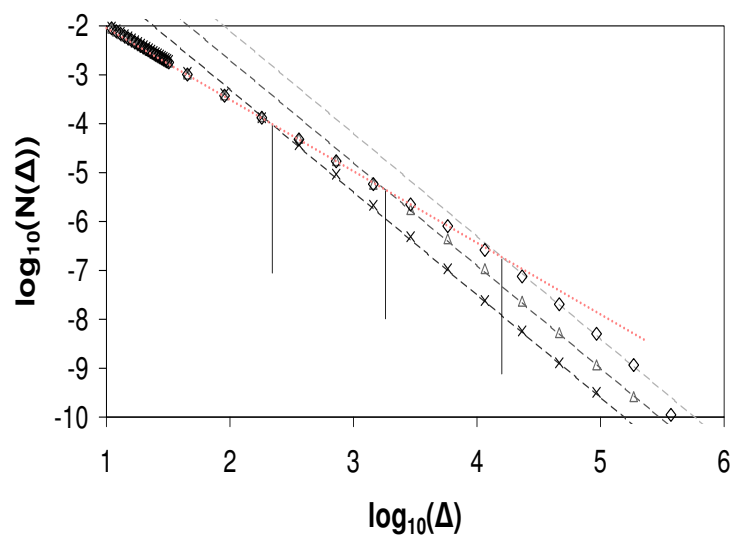

Figure 9. Avalanche distributions for the simplified model of imposed beam deformation (eqn (28)) for different mechanical properties of beam and fibers. Namely, the avalanche distributions for elastic beam $E I=12 \times 10^{10}$ and elastic fibers $k=1,(\triangle)$, for viscoelastic beam $\left(E I=12 \times 10^{10}, E / M=10^{4}\right)$ and elastic fibers $k=1,(\times)$, and for elastic beam $E I=12 \times 10^{10}$ and viscoelastic fibers $\left(k=1, k / \mu=10^{4}\right),(\diamond)$. The dashed lines are guidelines with slope -2.05 and the dotted one with slope -1.5 .

is equal to the corresponding values of the exact solution of system $(\Sigma)$. Omitting the oscillatory component one retrieves

$$
\left\{\begin{array}{l}
y^{\prime}(x, t)=A(t) e^{(\alpha \xi t-x) / \xi} \\
F^{\prime}(x, t)=\frac{\alpha k}{\frac{k}{\mu}+\alpha} A(t) e^{(\alpha \xi t-x) / \xi}
\end{array}\right.
$$

For initial conditions

$$
\left(y(x, 0)=A(0) e^{-x / \xi}, F(x, 0)=\frac{\alpha k}{\frac{k}{\mu}+\alpha} A(0) e^{-x / \xi}\right),
$$

expression (32) solves the problem of a wedge moving along $x$-axis with a time horizontal displacement of $U^{*}(t)=\xi(\alpha t+\ln A(t))$. This asymptotic solution has the same statistical properties with the simplified version of the model (28) derived in the previous section. Indeed, as it was pointed out in [11], the avalanche distribution of (32) depends solely on $\xi$ and it is independent of $U^{*}$. One may also come to the same conclusions for the case of initial conditions (24).

\section{Conclusions}

A model describing the crack propagation at the interface between a rigid substratum and a beam has been considered. The interface is modeled using a fiber bundle model (i.e. using a discrete set elements having a random strength). The distribution of avalanches, defined as the distance over which the crack is propagated under a fixed force, has been studied in order to capture correlations of breaking events in the course of fracture. Fiber breakage kinetics is related to a correlation length, which sets the size of the fracture process zone that occurs ahead of the crack due to progressive failure.

Ageing is considered as a variation of porosity of the interface. It corresponds for instance to diffusion controlled dissolution processes in cementitious materials 
(e.g. calcium leaching). Results obtained in [11] and described in the present paper show that the size of the fracture process zone is proportional to a length scale. This length scale increases (so does the size of the FPZ) upon increasing porosity and the discrete model is consistent with experiment data and numerical analysis.

The creep - fracture interaction has been analyzed in the second part of the paper. A simplified model is proposed, which is very similar to the static model used in the first part, but with a length scale that depends now on the time dependent characteristics of viscoelastic fibers and viscoelastic beam. It has been found that the size of the process zone depends on the fracture propagating velocity and on the distribution of forces in the interface due to the interaction between the interface and the rest of the specimen. The observed decrease of the size of the process zone, in creep experiments, compared to the size of the process zone in a static process, is justified by the proposed model for an interface that is less viscous than the rest of the material. This condition is realistic since the fibers should remain alive when the matrix material in between has failed, thus the proposed model is consistent with experimental evidence.

Acknowledgments. We thank A. Delaplace for providing us the numerical code used in [11]. This work was partially supported from the EU project 'Degradation and Instabilities in Geomaterials with Application to Hazard Mitigation' (DIGA-HPRN-CT-2002-00220) in the framework of the Human Potential Program, Research Training Networks.

\section{REFERENCES}

[1] J. Andersen, D. Sornette, and K.-T. Leung. Tricritical behavior in rupture induced by disorder. Physical Review Letters, 78(11):2140-2143, March 1997.

[2] G. G. Batrouni, A. Hansen, and J. Schmittbuhl. Heterogeneous interfacial failure between two elastic blocks. Physical Review E, 65:036126, 2002.

[3] Z. P. Bažant and R. Gettu. Rate effects and load relaxation: static fracture of concrete. ACI Materials Journal, 89(5):456-468, 1992.

[4] Z. P. Bažant and M. Jirasek. Nonlocal integral formulations for plasticity and damage: Survey of progress. Journal of Engineering Mechanics, 128:11191149, 2002.

[5] Z. P. Bažant and Y. N. Li. Cohesive crack with rate-dependent opening and viscoelasticity: I. mathematical model and scaling. International Journal of Fracture, 86:247-265, 1997.

[6] Z. P. Bažant and G. Pijaudier-Cabot. Nonlocal continuum damage, localization instability and convergence. Journal of Applied Mechanics ASME, 55:287-294, 1988.

[7] Z. P. Bažant and G. Pijaudier-Cabot. Measurement of the characteristic length of nonlocal continuum. Journal of Engineering Mechanics, 115:755-767, 1989.

[8] Z. P. Bažant and J. Planas. Fracture and size Effect in Concrete and Other Quasi-Brittle Materials. Boca Raton and London, 1998.

[9] H. Daniels. The statistical theory of the strength of bundles of threads. Proc. Royal Soc., A 183:405-435, 1945.

[10] A. Delaplace. Analyse Statistique de la Localisation dans les Matériaux Hétérogènes Quasi-Fragiles. PhD thesis, Ecole Normale Suprieure de Cachan, 1999.

[11] A. Delaplace, S. Roux, and G. Pijaudier-Cabot. Avalanche statistics of interface crack propagation in fiber bundle model: Characterization of cohesive crack. Journal of Engineering Mechanics, 127:646-652, July 2001. 
[12] K. Haidar, G. Pijaudier-Cabot, J.-F. Dubé, and A. Loukili. Correlation between internal length, fracture process zone and size effect in mortar and model materials. Materials and Structures, 38:201-210, 2005.

[13] D. G. Harlow and S. L. Phoenix. Approximations for the strength distribution and size effect in an idealized lattice model of material breakdown. Journal of the Physics and Mechanics of Solids, 39:173-200, 1991.

[14] A. Hillerborg, M. Modeer, and P. E. Pertersson. Analysis of crack formation and crack growth in concrete by means of fracture mechanics and finite elements. CCR, 6:773-782, 1976.

[15] C. Le Bellégo, J.-F. Dubé, G. Pijaudier-Cabot, and B. Gérard. Calibration of nonlocal damage model from size effect tests. European Journal of Mechanics, A/Solids, 22:33-46, 2003.

[16] C. Le Bellégo, G. Pijaudier-Cabot, B. Gérard, J.-F. Dubé, and L. Molez. Coupled chemical and mechanical damage in calcium leached cementitious structures. Journal of Engineering Mechanics, 129:333-341, 2003.

[17] C. Mazzotti and M. Savoia. Experimental study of nonlinear creep of concrete at high stress levels. In Z.P. Bažant, F.-J. Ulm, and F.H. Whitmann, editors, Proc. Concreep 6, pages 259-264, Cambridge, England, 2001. Elsevier, Amsterdam.

[18] W. I. Newman and S. L. Phoenix. Time-dependent fiber bundles with local load sharing. Physical Review E, 63(2):021507, 2001.

[19] G. Pijaudier-Cabot and A. Benallal. Strain localization and bifurcation in a nonlocal continuum. International Journal of Solids and Structures, 30:1761$1775,1993$.

[20] G. Pijaudier-Cabot, M. Omar, A. Loukili, and Y. Le Pape. Creep-damage interaction in concrete structures. In Proceedings of ICF 11, Torino, March 2005.

[21] S. Zapperi, P. Ray, H. E. Stanley, and A. Vespignani. First order transition in the breakdown of disordered media. Physical Review Letters, 78(8):1408-1411, March 1997.

[22] S.-D. Zhang, Z.-Q. Huang, and E.-J. Ding. Complex fiber bundle model for optimization of heterogeneous materials. Physical Review E, 54:3314-3319, 1996.

ERT R\&DO, Institut de Recherche en Génie Civil et Mécanique, Centrale Nantes, CNRS, Université de Nantes, 1 Rue de la nö̈, BP 92101, 44321 Nantes Cedex 3

E-mail address, Theocharis Baxevanis: theocharis@tem.uoc.gr

E-mail address, Frédéric Dufour: Frederic.Dufour@ec-nantes.fr

E-mail address, Gilles Pijaudier-Cabot: Gilles.Pijaudier-Cabot@ec-nantes.fr 